\title{
EJEMPLOS DE ANOMALÍAS DEL RADÓN 222 COMO PRECURSORAS DE EVENTOS SÍSMICOS EN COSTA RICA.
}

\author{
J. R. García-Vindas, Luis G. Loría M. \& Ricardo Jiménez D. \\ Universidad de Costa Rica, Escuela de Física, Sección de Física Nuclear Aplicada \\ Email: jrgarcia@cariari.ucr.ac.cr
}

(Recibido 16/6/1999; Aceptado 1/10/1999)

\begin{abstract}
The relation between seismicity and Radon -222 concentration anomalies has been studied. The changes in the gas concentration are considered as precursors of the seismic events. This study illustrates the before mentioned relation and confirms that aquifers are favorable places for radon monitoring.
\end{abstract}

RESUMEN: La relación entre la sismicidad y las anomalías de la concentración de Radón - 222 en el suelo ha sido estudiada. Las variaciones en la concentración de este gas se consideran como precursores de los eventos sísmicos. El presente estudio ilustra algunos ejemplos de la mencionada relación y a su vez confirma que los acuíferos son sitios favorables para el monitoreo de este gas. Las anomalías encontradas en los espectros de radón en los acuíferos de Carazo (Tres Rios, Cartago) y Puente Mulas (San Antonio de Belén, Heredia), así como las encontradas en los espectros tomados en Desamparados (San José), están asociadas a sismos cuyo hipocentro se localiza cercano a la estación de medida.

\section{INTRODUCCIÓN}

Los grandes desastres causados por los eventos sísmicos a lo largo y ancho de la Tierra han obligado a los científicos a buscar métodos mediante los cuales sea factible la predicción de tales sismos. Varios autores encuentran que las variaciones de ciertos parámetros geofísicos y geoquímicos de sistemas geológicos como acuíferos y fallas, presentan una fuerte correlación con la actividad sísmica.

Entre los cambios observados en diferentes sistemas geológicos antes, durante y después de un sismo se pueden citar:

a)- Variaciones en la concentración de iones en el agua antes y después del sismo (Hamond, 1981; King et al., 1981; Igarashi \& Wakita., 1995).

b)- Variaciones en la concentración de helio, hidrógeno y oxígeno (Reimer, 1981; Sugisaki 1984; O’ Neil, 1981), así como en las razones (He/Ar), (He/CH4) y $(\mathrm{CH} 4 / \mathrm{Ar})$ (Nagamine, 1994).

c)- Variaciones del orden de metros en el nivel freático (Merifield \& Lamar, 1981).

d)- Variaciones del flujo de agua dulce en fuentes naturales (King et al, 1994), incluyendo la desaparición de la fuente (Steele, 1981).

e)- Erupciones de agua asociadas con géiseres antes de un sismo (Wakita et al., 1988).

f)- Variación de la concentración de uranio y de la razón isotópica (234U/236U) antes de un sismo (Finkel, 1981).

En la naturaleza, en las cadenas de desintegración de ${ }^{238} \mathrm{U},{ }^{232} \mathrm{Th}$ y ${ }^{235} \mathrm{U}$ se hace presente en cada una de ellas un gas radiactivo conocido como radón, cuyas masas atómicas en su orden son 222, 220 y 219.

El ${ }^{222} \mathrm{Rn}^{\prime}\left(\gamma_{1 / 2}=3,82 \mathrm{~d}\right)$ se utiliza como trazador geoquímico en la predicción de eventos paroxísticos, por su alta movilidad, previa a su desintegración (Fleisher \& Mogro-Campero, 1979). 
El movimiento del gas es causado por los cambios en las tensiones de compresión y expansión en la zona sísmica. Estos cambios ocasionan el movimiento de ciertos fluidos (gases, agua, vapor) que transportan el radón hacia la superficie del suelo o hacia las capas internas de la Tierra, provocando, en ambos casos, una anomalía en la concentración de este gas en el subsuelo (Birchard, 1980; Fleisher, 1997; King, 1980; Steele, 1981). Estos dos tipos de movimiento son ilustrados en las figuras $1 \mathrm{a}$ y $1 \mathrm{~b}$. durante un período de actividad sísmica. En estas figuras se observa la variación de la concentración de radón cerca de la superficie del suelo (a un metro de profundidad) en varias estaciones, asociadas con una crisis sísmica. Si bien en las figuras 1 a y 1 b las anomalías en la concentración de radón son asociadas con eventos de magnitud mayor o igual que 7, esta correlación puede también encontrarse con eventos de baja magnitud (King, 1980; Martinelli et al., 1995; Notsu et al., 1991) especialmente si se trata de una sismicidad local, donde las anomalías pueden ser asociadas hasta con sismos de magnitud igual a 2 .
Si bien las anomalías son interpretadas como precursoras de eventos mayores, la interpretación de los datos que se obtienen es bastante compleja, pues existen diversos parámetros externos que influyen en la emanación de este gas y por lo tanto su concentración en el suelo. Estas variables son principalmente la lluvia, la presión atmosférica, temperatura y la humedad relativa del suelo, (Clements \& Wilkening, 1974; Klusman \& Jaacks, 1987; Abbad et al., 1996; Talwani et al., 1980). En nuestro país el principal factor que produce variaciones importantes en la concentración de radón es la lluvia, pues los cambios producidos por los otros parámetros son despreciables, ya que la variación máxima, de la presión atmosférica es de 2 mbares en un período de 12 horas (V. Castro, com. pers., 1998). Las variaciones de temperatura atmosférica son de 5 grados durante el mismo período, por lo que no es factible que se puedan crear celdas de convección a una profundidad de un metro. El presente trabajo muestra un estudio de la variación de la concentración de radón en diferentes estaciones de monitoreo localizadas en las fuentes de agua

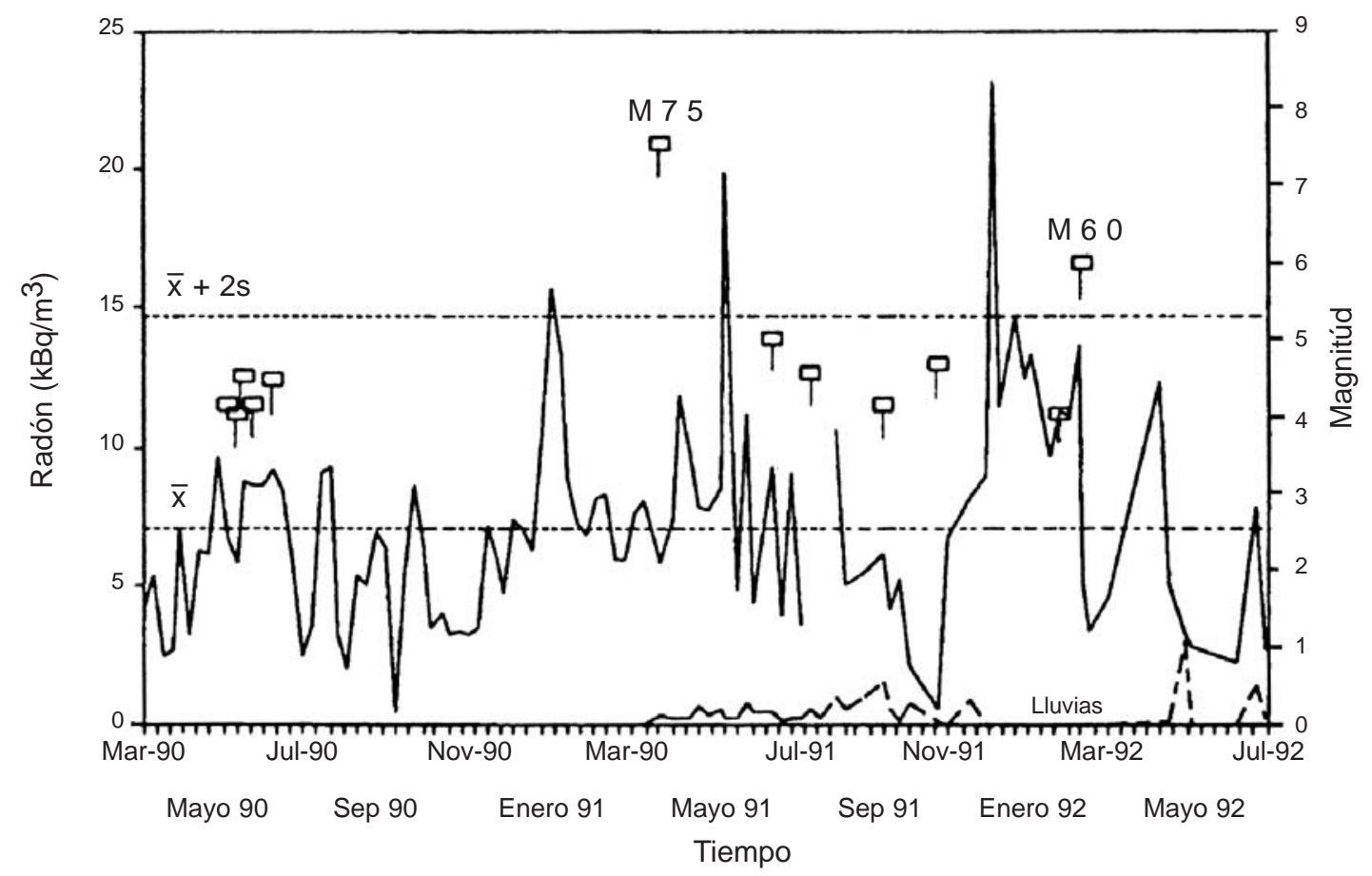

Fig. 1-a: Variación temporal del Radón y la actividad sísmica (Loría et al., 1995). 


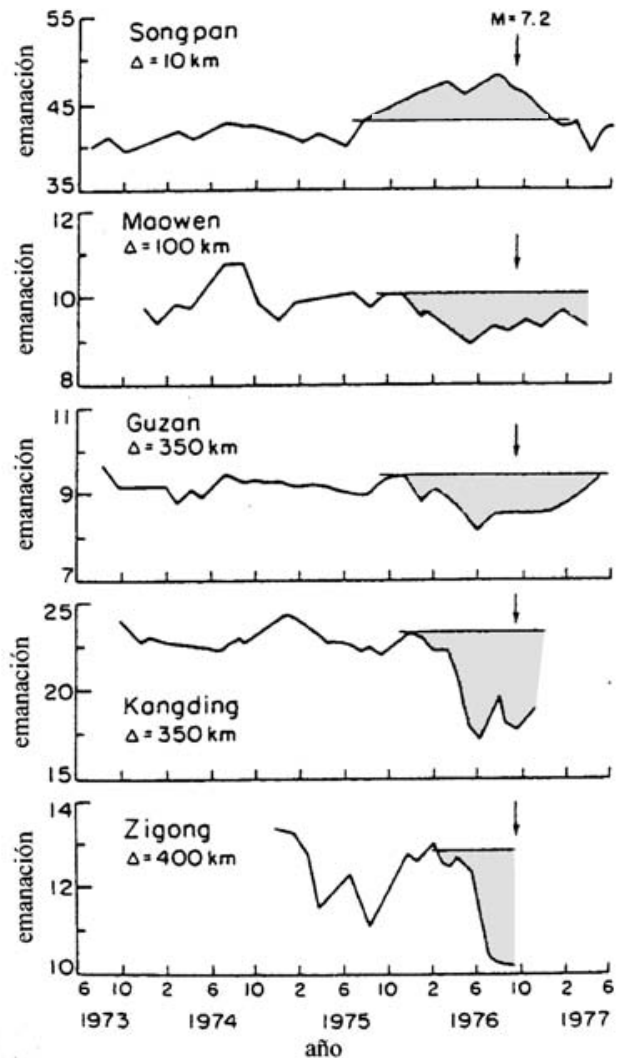

Fig. 1-b: Medida de la concentración de radón efectuada en diferentes estaciones, durante el sismo de Songpan, Japón. El símbolo D representa la distancia entre la estación y el epicentro (Fong-liang \& Gui-ru, 1981).

de San Antonio de Belén (Puente de Mulas), Tres Ríos (fuente de Carazo), la falla Salitrillos que atraviesa San Antonio de Patarrá y San Miguel de Desamparados, y su posible correlación con la sismicidad local y regional.

\section{MATERIALES Y MÉTODOS}

La detección del gas radón se realiza a través de la emisión de partículas producto de su desintegración. Esta partícula tiene carga positiva y es registrada en general con un detector de barrera superficial a base de silicio.

El dispositivo utilizado en la medición del gas consta esencialmente de un cristal de silicio, de un módulo que amplifica y discrimina la señal, y de otro de memoria que contabiliza y registra la señal. Este dispositivo conocido como Clipperton II (Monnin \& Seidel, 1998), integra las lecturas de las señales durante un paso de tiempo programado previamente por el usuario. Este paso de tiempo se escoge para períodos de lectura que oscilan desde un minuto hasta 48 horas.

La programación de la sonda se lleva a cabo con una interface Psion LZ-64, la cual a su vez permite leer las series de tiempo y almacena la información en una memoria Eprom, para luego ser transferidas a un ordenador personal.

El dispositivo es colocado en un hueco recubierto con PVC con una profundidad de un metro. El espacio entre la sonda de lectura y el extremo superior del tubo es llenado con un material aislante para evitar la formación de celdas de convección en ese espacio, las cuales permiten flujos de gas que alteran la medida del radón. El cristal de silicio que recoge la señal de radón queda expuesto a $10 \mathrm{~cm}$ del fondo del agujero. $\mathrm{La}$ distancia fondo cristal ha sido calibrada de tal manera que no haya lectura importante en él (menos del $0,05 \%$ ) de las emisiones alfa de otros isótopos del radón (García-Vindas, 1999). En la figura 2 se muestra la instalación de la sonda en una de las estaciones de medición terrenas.

Cuando las mediciones se realizan en acuíferos, la sonda está sujeta por un dispositivo que mantiene la distancia de separación entre el

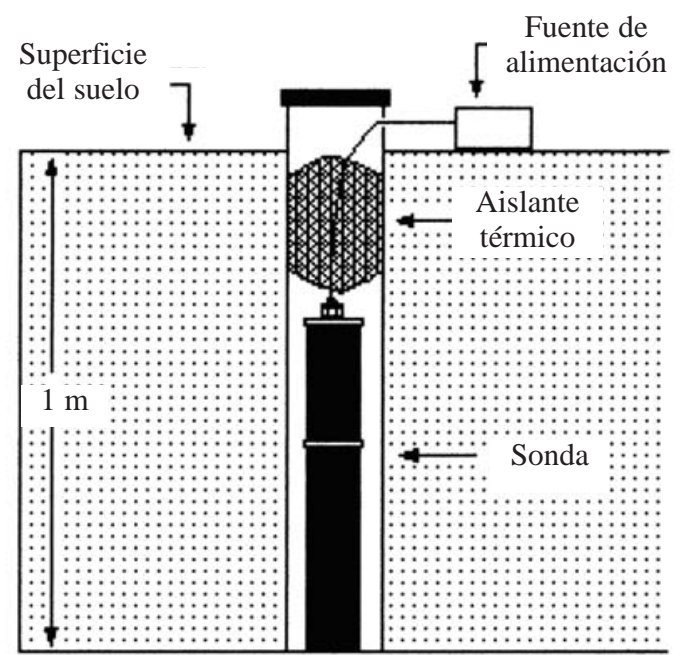

Fig. 2: Diagrama de instalación de la Sonda Clipperton II en el campo (Monnin \& Seidel, 1998). 
cristal y la superficie del agua constante. La sonda se instala a la salida del acuífero de tal manera que el radón que llega a la sonda proviene exclusivamente del agua.

Las medidas de la concentración de radón provenientes de los sitios monitoreados se recogen con un paso de lectura de 1 hora, para hacerlos coincidir con los datos meteorológicos tomados en ese mismo intervalo de tiempo y de esta forma establecer si se trata de una señal precursora o una distorsión provocada por estos parámetros.

\section{RESULTADOS}

Los resultados que se muestran a continuación, han sido seleccionados de un gran conjunto de datos, correspondientes con mediciones de la concentración de radón que han sido obtenidos durante un período de varios años. Estos muestran una correlación evidente entre la señal de radón y la actividad sísmica en ese momento.

\section{Acuífero de Puente de Mulas}

La señal de radón en esta estación, en los meses previos a febrero de 1997, presenta sólo variaciones estadísticas, pero durante el mes en mención, el espectro muestra un aumento en la concentración promedio del gas, la cual concuerda con la actividad sísmica registrada entre el 13 y 25 de febrero de 1997.

Los hipocentros de los sismos que se indican en la figura se encuentran localizados a 170, 40 y 8 km de la estación (Erick Fernández, OVSICORI, com. pers., 1997).

En la Figura 3 se muestra el espectro de emisión de radón en esta estación, correspondiente al mes de febrero de 1997, que corresponde al período seco, esto es no hay presencia de precipitación en este mes, por lo que no existe influencia de la lluvia sobre la concentración del radón.

\section{Acuífero de Carazo}

En esta estación, alrededor de los días 20 y 21 de setiembre de 1997, la concentración del gas aumentó aproximadamente 3 veces con respecto a las mediciones de los días anteriores, para luego volver a sufrir sólo variaciones estadísticas. El aumento en la concentración, al igual que lo observado en Puente de Mulas, muestra una correlación evidente con los sismos

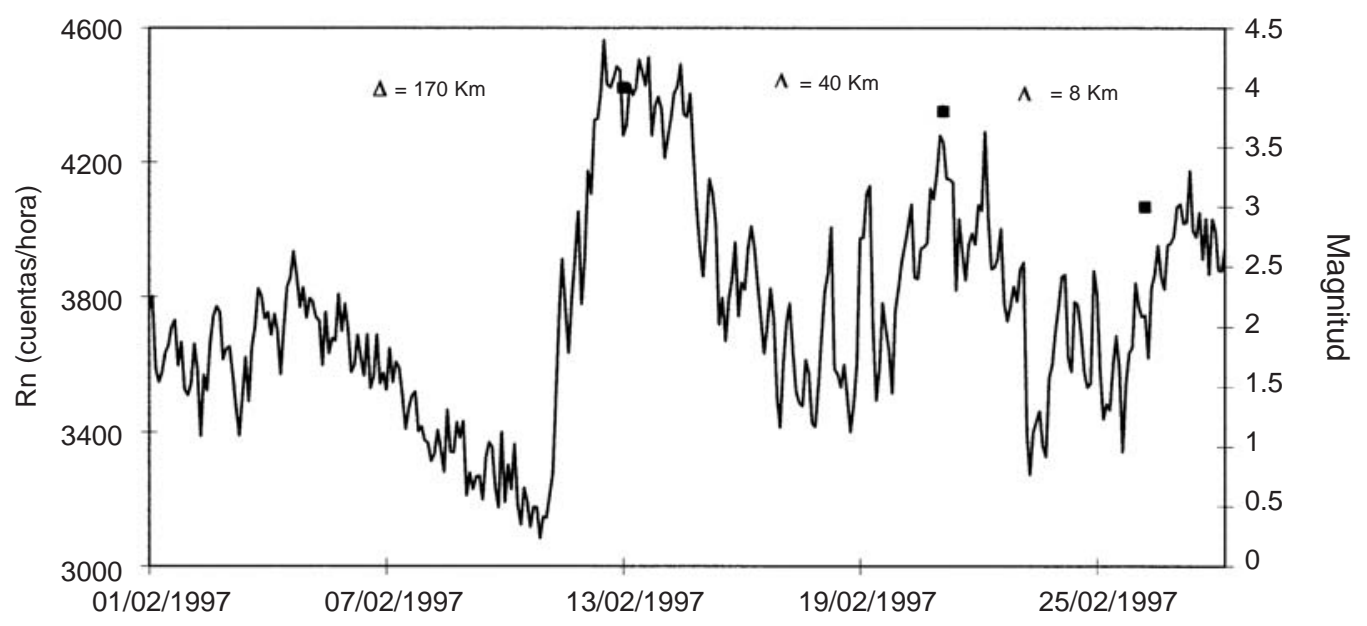

Fig. 3: Variación de la concentración de radón durante un período de actividad sísmica. Acuífero de Puente de Mulas (los cuadrados indican la ocurrencia del sismo y el símbolo D la distancia al hipocentro). 


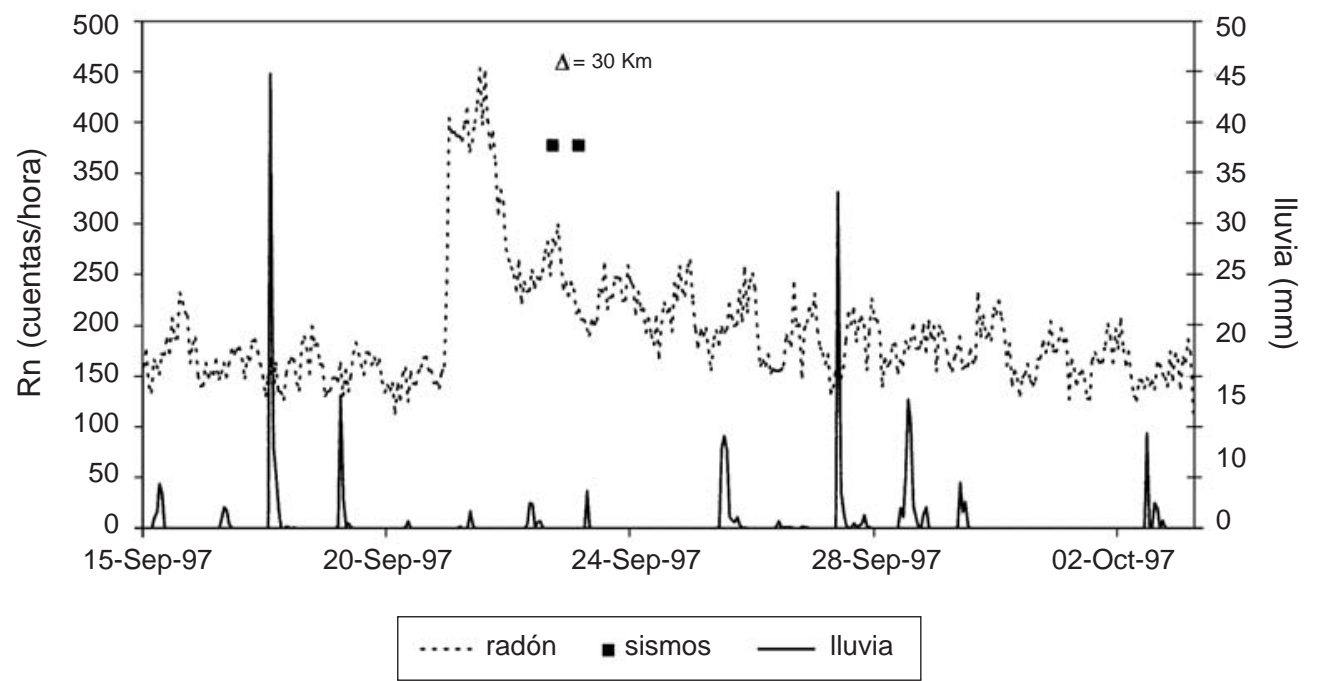

Fig. 4: Variación de la concentración de radón y de la precipitación durante un período de actividad sísmica. Fuente de Carazo (los cuadrados indican la ocurrencia del sismo y el símbolo D la distancia al hipocentro).

del día 23 de ese mes, los cuales tienen su hipocentro a $30 \mathrm{~km}$ del acuífero, con una magnitud de 3,0 en la escala Richter (Erick Fernández, OVSICORI, com. pers., 1997). En la figura 4 se presenta el espectro de emisión del gas durante la segunda mitad del mes de setiembre del año de 1997, así como la precipitación del mismo período. En esta figura se observa que la precipitación es escasa entre los días 18 y 27 de ese mes con lo que un posible efecto de esta sobre el gas sería de poca importancia.

\section{Falla de Salitrillos}

Dos de las tres sondas instaladas sobre esta falla en agosto de 1997, muestran un aumento en la concentración del gas días antes de los sismos ocurridos el 9 de mayo de 1998, con una magnitud de 3,8 grados en la escala Richter y cuyo hipocentro está ubicado a $80 \mathrm{~km}$ de la estación de medición. De igual manera la concentración del gas presenta la misma anomalía para los sismos del 17 de julio de 1998, los cuales ocurrieron a $200 \mathrm{~km}$ de la estación, con magnitudes de 5,2 y 4,2 en la escala Richter. Al igual que en los acuíferos la concentración del gas aumenta en promedio tres veces con relación a las mediciones previas. En la figura 5 se presenta el espectro de emisión del gas y los sismos asociados con la anomalía del gas en los meses de abril y mayo de 1998 y en la figura 6, la evolución de la concentración durante los meses de junio, julio y principios de agosto del mismo año.

\section{CONCLUSIONES}

Los resultados obtenidos muestran que el equipo utilizado permite observar variaciones de corto período en la concentración de radón, las cuales no se observarían al utilizar detectores plásticos, dado que estos integran la señal en períodos mucho mayores, que aquellos en que se producen las variaciones. Además la metodología y el equipo empleado en los dos tipos de sitio, para la recolección de los espectros de radón, indican que estos son los adecuados para el monitoreo de este gas.

En todos los casos la correlación entre la sismicidad y las anomalías en la concentración de 


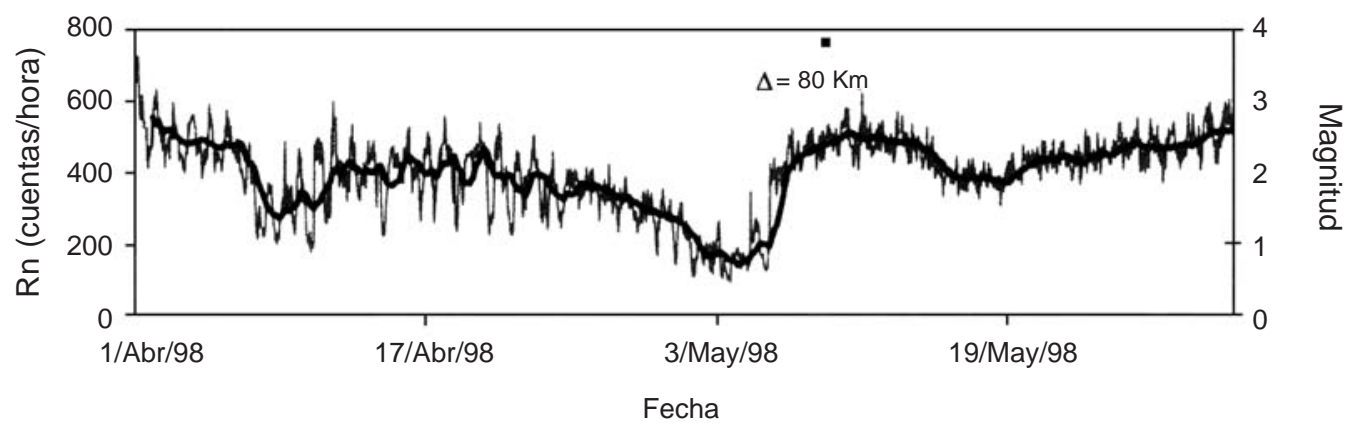

a-

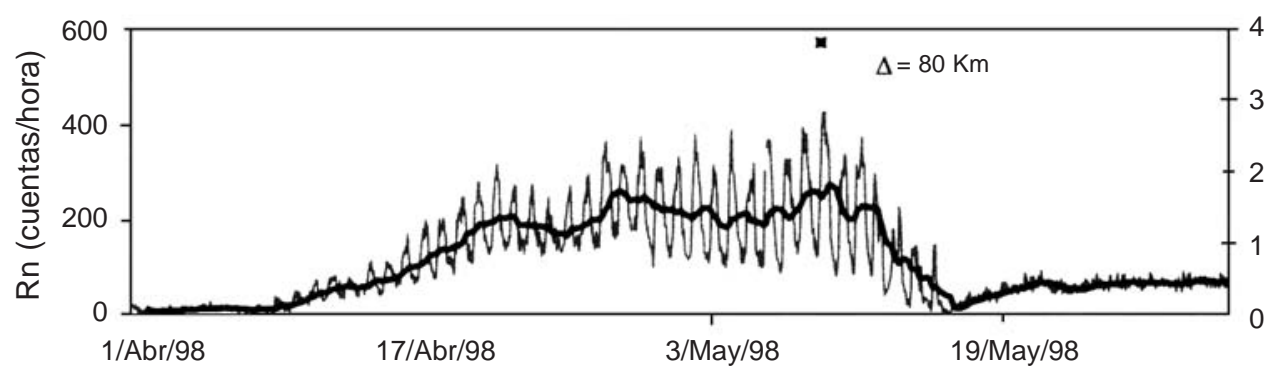

일

b-

Fecha

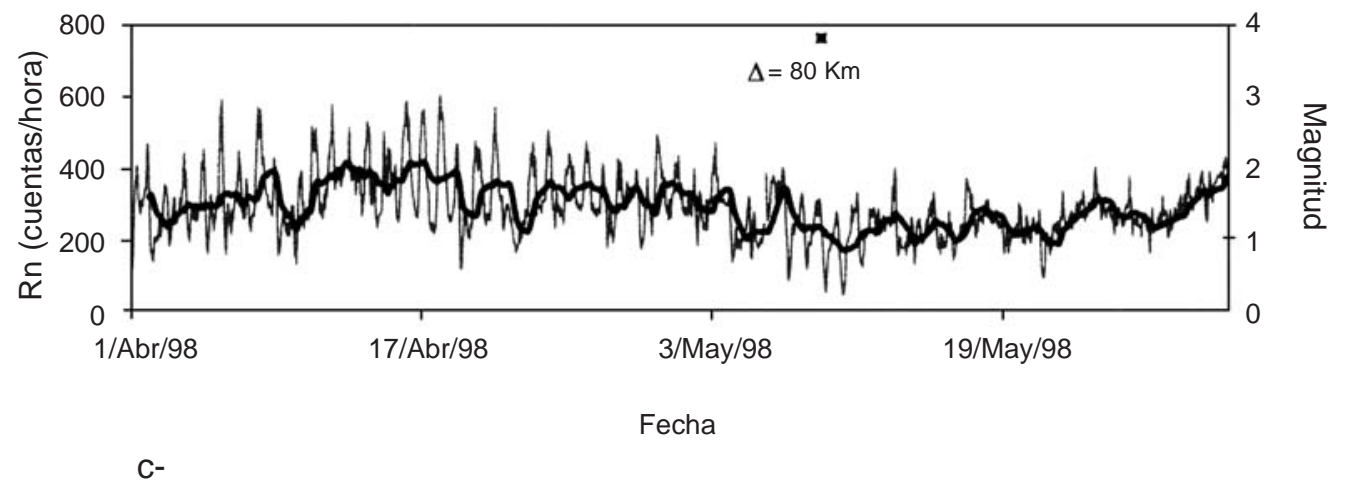

Fig. 5: Evolución de la concentración de radón en tres sondas instaladas en la Falla de Salitrillos. En línea gruesa se presenta la curva de tendencia cuando se calcula la media móvil con un período de 24 horas (los cuadrados indican la ocurrencia del sismo y el símbolo D la distancia al hipocentro). 


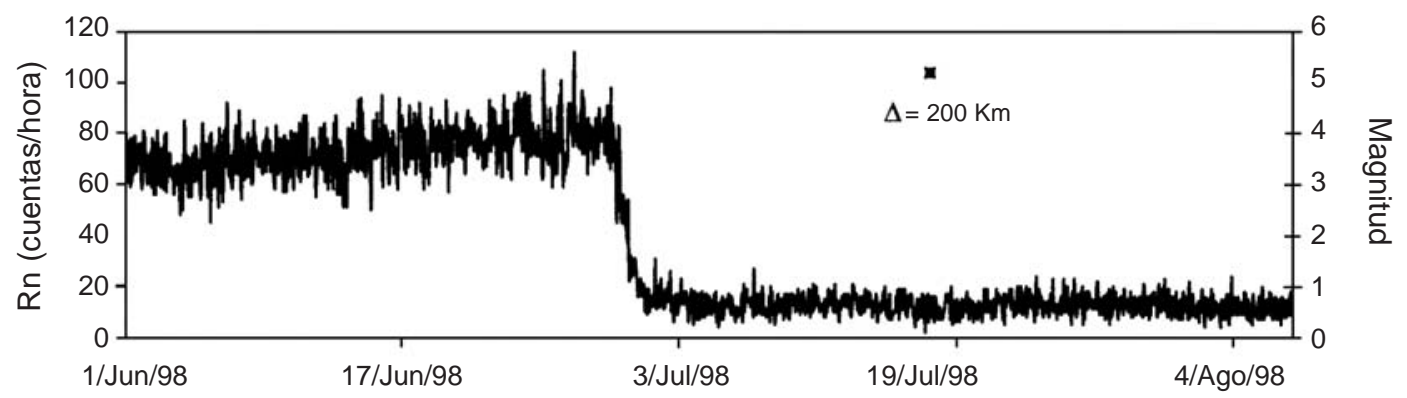

a-

Fecha

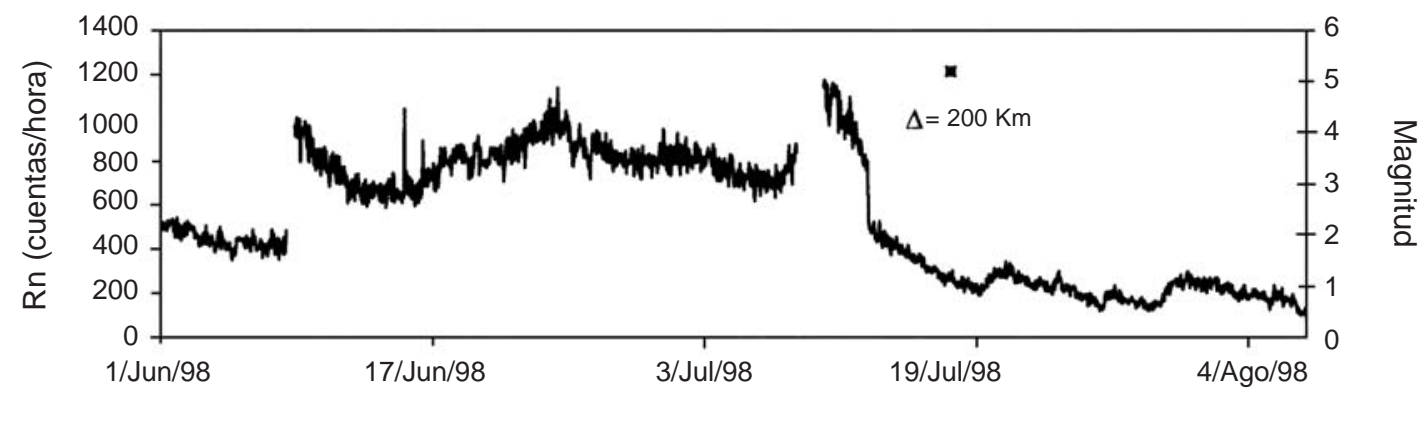

b-

Fecha

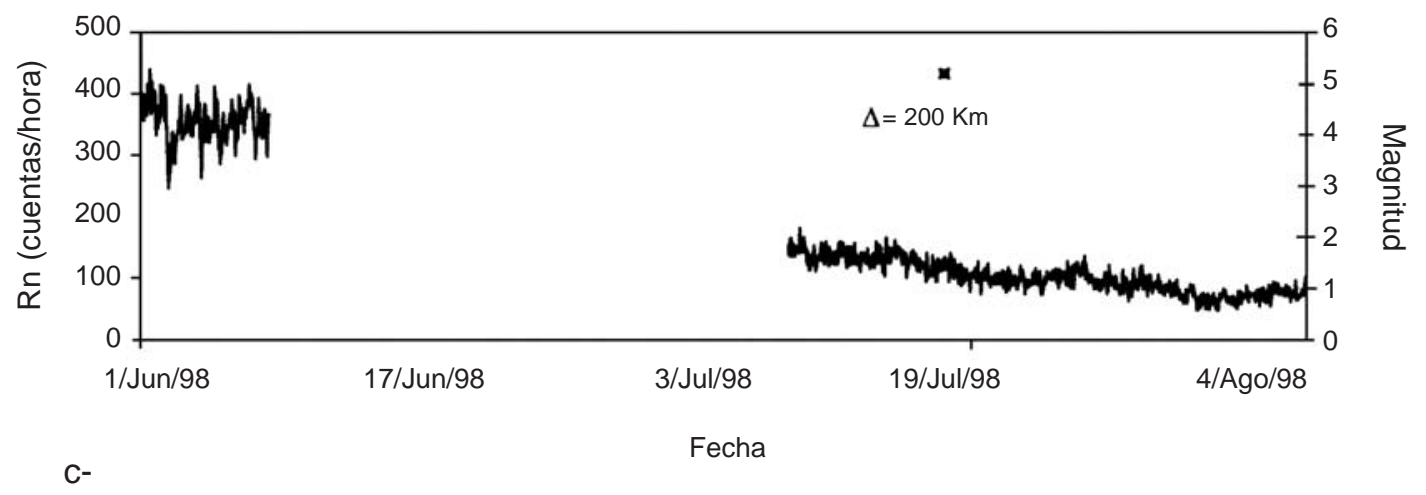

Fig. 6: Evolución de la concentración de radón en tres sondas instaladas en la Falla de Salitrillos (los cuadrados indican la ocurrencia del sismo y el símbolo D la distancia al hipocentro). 
radón esta de acuerdo con el modelo expuesto por Fleisher (1981), el cual establece una relación empírica entre la magnitud del sismo y la distancia promedio entre el epicentro y la anomalía percibida.

Los resultados de este estudio sugieren mantener un constante monitoreo de este gas y de los parámetros exteriores que lo afectan; principalmente en las zonas más activas del país, realizando un análisis más frecuente de los datos que se obtienen. Esto contribuiría a aumentar la probabilidad de encontrar anticipadamente las señales precursoras de algunos de los eventos sísmicos.

Las anomalías observadas en la concentración de radón en los acuíferos estudiados, concuerdan con las observadas por otros autores (Loría et al., 1995; King, 1980; Fong-liang y Guiru, 1981), ante el advenimiento de un sismo, lo que permite inferir que estos sitios son los ideales para monitorear el gas y correlacionar los espectros de emisión del mismo con la sismicidad.

El hecho de que una de las sondas de Desamparados no muestre cambios importantes en el espectro de emisión a raíz de los sismos del 9 de mayo y 17 de julio de 1998, podría ser un indicador de que los efectos producidos por la actividad sísmica sobre la falla son menos importantes en esta zona. Este mismo efecto ha sido encontrado por otros autores (King, 1984). Lo anterior obliga a realizar un estudio detallado de las características del suelo, entre ellas porosidad y permeabilidad antes de la instalación de un equipo de medición.

Los ejemplos presentados de anomalías en el espectro de emisión del radón y que se relacionan con sismos, obliga a continuar con el monitoreo de éste con el fin de encontrar correlaciones que permitan en un futuro cercano la predicción de los mismos, especialmente los de mayor magnitud y de esta forma preparar a la población, como también evitar la pérdida de vidas humanas.

\section{AGRADECIMIENTO}

Los autores queremos dejar patente nuestro agradecimiento a la Universidad de Costa Rica, en especial a la Vicerrectoría de Investigación por el apoyo brindado, a través del proyecto "Radón y Sismicidad en Costa Rica". A la Universidad de Montpellier II e igualmente al Organismo Internacional de Energía Atómica.

\section{REFERENCIAS}

ABBAD, S., ROBE, M.C., BERNAT, M. \& LABED, V., 1996: Influence of meteorological and geological parameter variables on the concentration of radon in soil gases: application to seismic forecasting in the ProvenceAlpes-Cote d'Azur region.- Environmental Geochem. Health, 16: 35-48.

BIRCHARD, G.F. \& LIBBY, W.F., 1980: Soil Radon concentration changes preceding and following four magnitude 4.2-4.7 earthquakes on the San Jacinto fault in southern California - Geophys. Res. 85: 3100-3106.

CLEMENTS, W. \& WILKENING, M., 1974: Atmospheric pressure effects on Rn222 transport across the Earth-Air interface.Geophys. Res. 29: 5025-5029.

FINKEL, R.C., 1981: Uranium concentrations and U-234/U-238 activity ratios in fault-associated groundwater as possible earthquake precursors.- Geophys. Res. Lett.8: 453-456.

FLEISCHER, R.L. \& MOGRO-CAMPERO, A., 1979: Radon enhancements in the earth: evidence for intermittent upflows?.- Geophys. Res. Lett. 6: 361-364.

FLEISCHER, R. L., 1981: Dislocation model for radon response to distant earthquakes.Geophys. Res. Lett. 8: 477-480.

FLEISCHER, R.L., 1997: Radon: Overview of properties, origin, and transport. En: DURRANI, S.A., \& ILIC, R. (eds.): Radon measurements by etched track detectors: 320. - Saeed A. Durrani \& Radomir Ilic, World Sci. Publ. Singapore. 
FONG-LIANG, J. \& GUI-RU, L., 1981: The application of geochemical methods in earthquake prediction in China.- Geophys. Res. Lett. 8: 445-448.

GARCIA-VINDAS, J. R., 1999: Transport du radon en milieu poreux (experimentation et modelisation): implication pour la realisation et l'interpretation de mesures in situ. 271 págs. Université de Montpellier, Francia [Tesis PhD.].

HAMMOND, D.E., TENG, T.L., MILLER, L. \& HARAGUCHI, G., 1981: A search for covariance among seismicity, groundwater chemistry, and groundwater radon in Southern California.- Geophys. Res. Lett. 8: 445448.

IGARASHI, G. \& WAKITA, H., 1995: Geochemical and hydrological observations for earthquake prediction in Japan.- J. Phys. Earth, 43: 585-598.

KING, C.Y., 1980: Radon emanation in tectonically active areas.- Nat. Radiat. Environ: 178-183.

KING, C.Y., EVANS, W.C., PRESSER, T. \& HUSK, R.H., 1981: Anomalous chemical changes in well waters and possible relation to earthquakes.- Geophys. Res. Lett. 8: 425428.

KING, C.Y., 1984: Impulsive radon emanation on a creeping segment of the San Andreas Fault, California.- Pageoph. 122: 340-351.

KLUSMAN, R.W., \& JAACKS, J.A., 1987: Environmental influences upon Mercury, Radon and Helium concentrations in soil gases at site near Denver, Colorado.- J. Geochemical Explor. 27: 259-280.

LORÍA, L., BRENES, J., FERNANDEZ, E., JIMÉNEZ, R., GARCIA, R., MONNIN, M., SEIDEL, J.L., SEGOVIA, N. \& BALCAZAR, M., 1995: Radon and seismicity in
Costa Rica. - Environ. Geochem. Health, 16: 213-219.

MARTINELLI, G., ALBARELLO, D. \& MUCCIARELLI, M., 1995: Radon emissions from mud volcanoes in Northern Italy: possible connection with local seismicity.Geophys. Res. Lett. 22: 1989-1992.

MERIFIELD, P.M. \& LAMAR, D.M., 1981: Anomalous water-level changes relation with earthquakes.- Geophys. Res. Lett. 8: 437-440.

MONNIN, M. \& SEIDEL, J., 1998: An automatic radon probe for earth science studies.- J. Appl. Geophys. 39: 209-220.

NAGAMINE, K., 1994: Origin and coseismic behavior of mineral spring gas at Byakko, Japan, studied by automated gas chromatographic analyses.- Chem. Geol. 114: 3-17.

NOTSU, K., WAKITA, H., IGARASHI, G. \& SATO, T., 1991: Hydrological and geochemical changes related to the 1989 seismic and volcanic activities of the Izu Peninsula.J. Phys. Earth, 39: 245-254.

O'NEIL, J.R. \& KING, C.Y., 1981: Variations in stable-isotope ratios of ground waters in seismically active regions of California.Geophys. Res. Lett. 8: 429-432.

REIMER, G.M., 1981: Helium soil-gas variations associated with recent Central California earthquakes: Precursor or coincidence? Geophys. Res. Lett. 8: 433-435.

STEELE, S.R., 1981: Radon and hydrologic anomalies on the rough creek fault possible precursors to the M5.1 Eastern Kentucky earthquake, 1980.- Geophys. Res. Lett. 8: 465468.

SUGISAKI, R., 1984: Relation between Hydrogen emission and seismic activities.- $\mathrm{Pa}$ geoph. 122: 175-184. 
TALWANI, P., MOORE, W.S. \& CHIANG, J., 1980: Radon anomalies and microearthquakes at Lake Jocassee, South Carolina.- J. Geophys. Res. 85: 3079-3088.
WAKITA, H., NAKAMURA, Y. \& SANO, Y., 1988, Short-term and intermediate-term geochemical precursors.- Pageoph. 126: 267-278. 